\title{
An overview of TEAM strategies for integrating the product realization process
}

\author{
C.K. Cobb \\ Lockheed Martin Energy Systems \\ P.O. Box 2009, MS-8160 \\ Oak Ridge, TN 37831 USA \\ Phone: (423) 576-1884 \\ Fax: (423) 574-4748 \\ cli@ornl.gov
}

\begin{abstract}
The Technologies Enabling Agile Manufacturing (TEAM) program enhances industrial capability by advancing and deploying manufacturing technologies that promote agility. TEAM is leveraging the expertise and resources of federal agencies, private industry, and universities to develop, integrate, and deploy leapahead technologies. TEAM has developed a product realization process that features the integration of product design and manufacturing groups, highlighted by optimization of product, process, and resource design parameters.

The TEAM Web Integration Manager (WIM) tool was developed to integrate this product realization process for the September 1997 integrated material removal demonstration, which featured the fabrication and inspection of a General Motors V8 cylinder head. The WIM provides comprehensive Web-based management of requirements, product attributes, process attributes, resources, and users. It managed the completion of process tasks from requirements definition through fabrication and inspection, ensuring controlled access to consistent design and manufacturing information. In addition, a specialized user interface into the WIM was deployed to provide detailed Web-based integration with a CAD system and with stress analysis, cost analysis, and optimization tools to provide a high level of automation in the Concept Optimization phase of the TEAM process.
\end{abstract}

Keywords

Agile manufacturing, virtual enterprise, product realization, enterprise integration, Web integration 
The Technologies Enabling Agile Manufacturing (TEAM) program (Neal, 1995) enhances industrial capability by advancing and deploying manufacturing technologies that promote agility. TEAM is leveraging the expertise and resources of federal agencies, private industry, and universities to develop, integrate, and deploy leap-ahead technologies. TEAM has developed a product realization process that features the integration of product design and manufacturing groups, highlighted by optimization of product, process, and resource design parameters.

The TEAM Web Integration Manager (WIM) tool was developed to integrate this product realization process for the September 1997 integrated material removal demonstration. The WIM provides comprehensive Web-based management of requirements, product attributes, process attributes, resources, and users. It managed the completion of process tasks from requirements definition through fabrication and inspection, ensuring controlled access to consistent design and manufacturing information. In addition, a specialized user interface into the WIM was deployed to provide detailed Web-based integration with a CAD system and with stress analysis, cost analysis, and optimization tools to provide a high level of automation in the Concept Optimization phase of the TEAM process.

This paper presents an overview of the TEAM product realization process and workflow process models. The Web-based tools deployed to integrate the process for the product design and analysis, process planning, simulation and verification, fabrication, and inspection of a General Motors V8 cylinder head are then described. The cylinder head was the product vehicle for the September 1997 demonstration.

More information on the TEAM program can be found at the URL http://cewww.eng.ornl.gov/team/home.html.

\subsection{The Product Realization Process}

The TEAM product realization process is derived from the TEAM product realization model (Figure 1) and is driven by customer needs to provide solutions in the form of products and services. It consists of Concept Optimization, Design Optimization, and Execution phases. This process is based on development and execution of a product realization script. The script, which is produced through close collaboration by all of the participants in the product realization process, contains all of the information needed to manufacture a product, stored in a format that makes it usable and readily available to all who need it. It also serves as a repository for all information about the product and its manufacturing process. The information in the script is optimized by trading off critical product, process, and resource parameters. The script then becomes the "master" for creating product during the execution phase, where acquisition, allocation, fabrication, and assembly are conducted to produce the end products. 


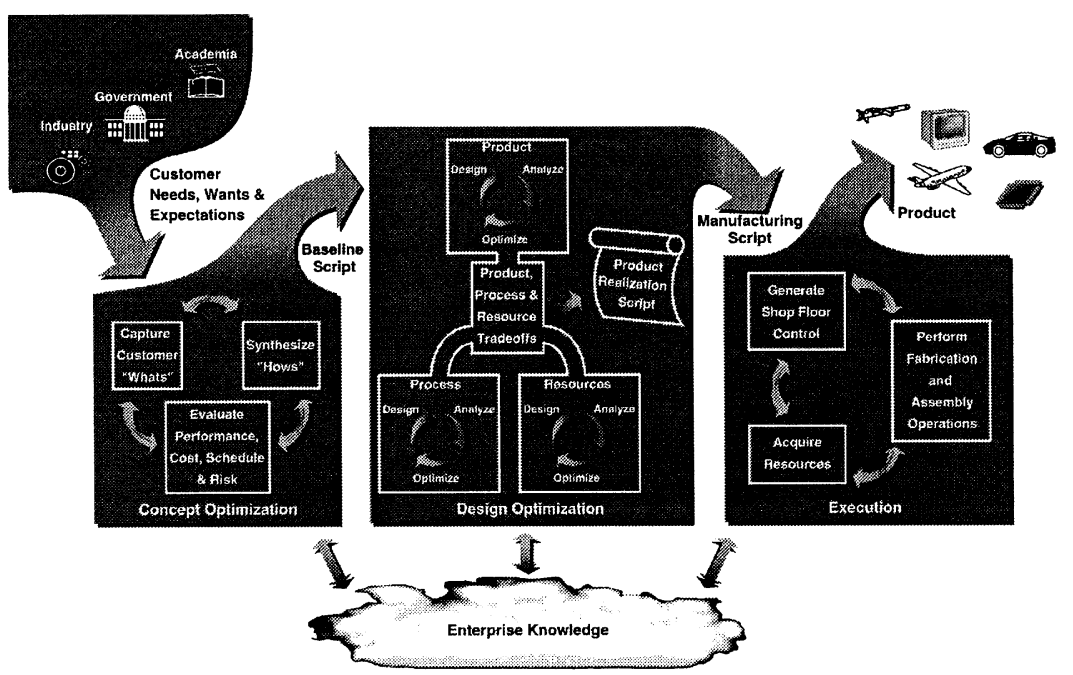

Figure 1 The TEAM Product Realization Model.

The product realization script is based on enterprise knowledge captured from past experience and includes information and domain knowledge for similar products, product models, manufacturing processes, and enterprise resources. These knowledge assets are integrated through an infrastructure that enables collaboration by different partners and interoperability of systems and tools.

Concept optimization is the first step in the TEAM product realization process. This step captures the customer's needs and desires, converts them into requirements, and translates the requirements into optimized concepts in the form of a baseline script. This is accomplished through an iterative process of capturing and prioritizing customer needs, defining solutions to those needs, establishing target parameters for the solutions, and analyzing and optimizing these parameters relative to design targets. This agreement between the customers and the providers is documented in the form of a product design and supporting information which are captured in the baseline script.

After the baseline script is in place, there is still more work to perform to create the detailed information needed to execute the manufacturing process. Detailed analyses that were not necessary to develop the initial product realization concepts must now be performed to optimize the designs of the product and its manufacturing processes. Manufacturing information that drives the factory must be created in the right format, and all of this information must be optimized for performance and cost-effectiveness. This is the Design Optimization phase, which translates a low-fidelity baseline script into a high-fidelity manufacturing script. The design optimization process involves three iterative design optimization environments: products, manufacturing processes, and enterprise resources.

The Execution phase implements the manufacturing script to produce tangible products. Necessary information is extracted from the manufacturing script to operate the enterprise's manufacturing processes and deliver the desired product. 


\subsection{The Workflow Model}

The product realization model provides a high-level view of the strategy for product realization. However, the strategy must be tailored for specific product requirements. This is accomplished using workflow models. They bring the right tools at the right time to do the right job in an integrated environment of total product and process optimization.

In the Concept Optimization phase (Figure 2), there is close interaction with the customer to capture the needs and desires and convert these into product, process, and resource requirements. For estimating and communication purposes, a parametric, features-based solids model of the product is created to identify the requirements, materials, and features of the product that drive its manufacturability, cost, and schedule. The overall deliverable from Concept Optimization is the baseline script, which consists of the functional requirements, "first cut" solids model of the product, process, and resource information, and design rationale. Detailed, high-fidelity decisions are deferred to the next phase in the process.

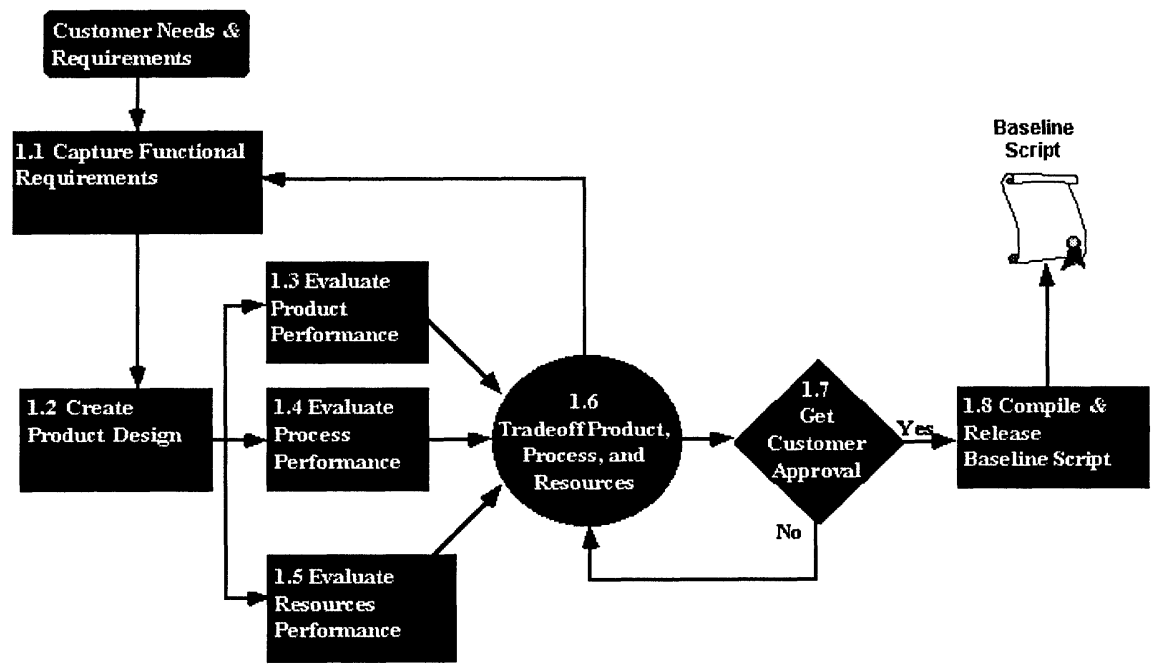

Figure 2 Concept Optimization Workflow.

Once customer needs are established to the point that the product's requirements can be clearly and completely defined, the functions of producibility, process modeling, simulation, analysis, and resource planning are conducted, interoperating seamlessly and concurrently, to provide accurate assessments of cost, performance, and schedule for various product realization approaches. In this process, all options are considered in a systematic way, not just to evaluate the impact of individual operations, but to assess their interaction with all other 
operations. This ensures that the best decisions are made. These processes enable the rapid tradeoffs of key factors so that an optimized, validated design may be determined for the product and its design and manufacturing processes.

This is all part of the Design Optimization phase (Figure 3). Simulation and optimization tools take the baseline script to the next level of detail to fine tune the best methods, materials, and processes for manufacturing execution. The manufacturing script is prepared during this step. The manufacturing script is the total package of information required to produce the product accurately, efficiently, on time, and within budget. It consists of the solids model, operations process plans, validated part manufacturing and inspection programs, and supporting work instructions.

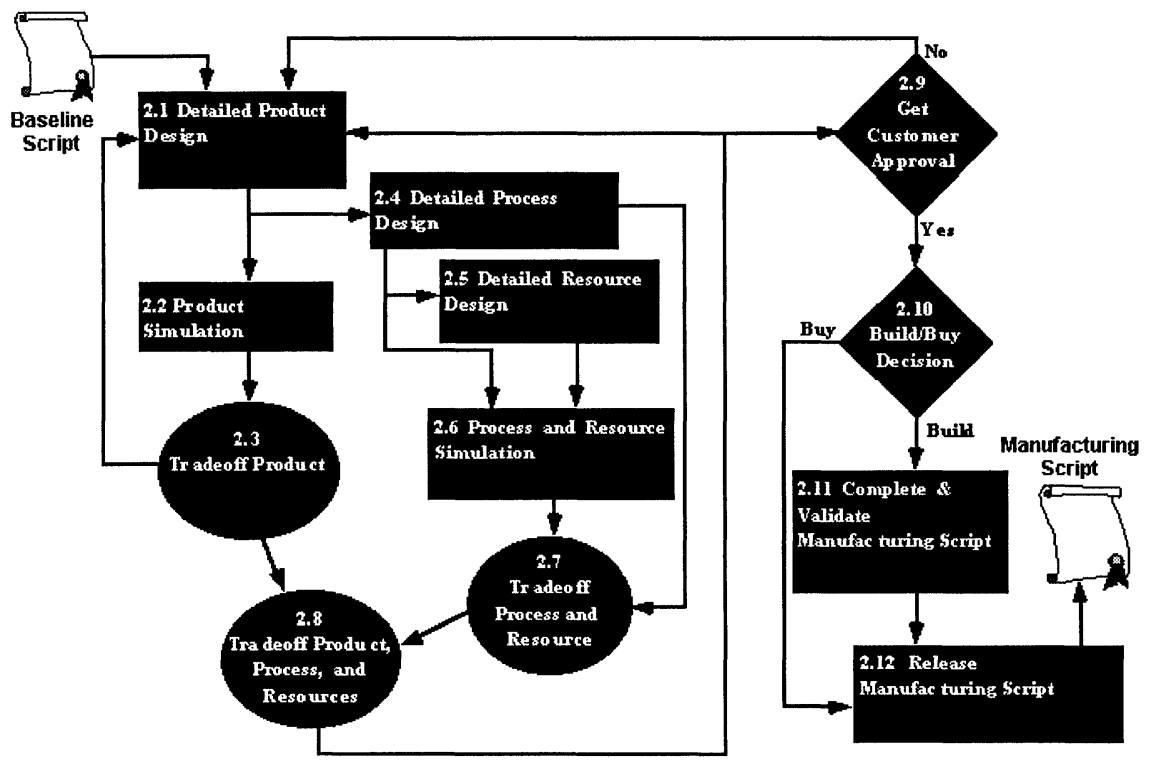

Figure 3 Design Optimization Workflow.

The Execution phase (Figure 4) of the product realization process is where the information generated by the process is put to use in making product. In this phase, intelligent closed-loop manufacturing processes are performed with 100\% assurance of a quality result. Such certainty is possible because control models are in place for each process and the parameters of each model are supplied in the manufacturing script for every product. The process control models are the result of a systematic process called deterministic manufacturing. This means that processes are characterized by defining all of their parameters, quantifying their interactions and impacts on process performance, defining cost and performance tradeoffs to define control limits, and developing monitoring and control strategies to ensure correct execution. 


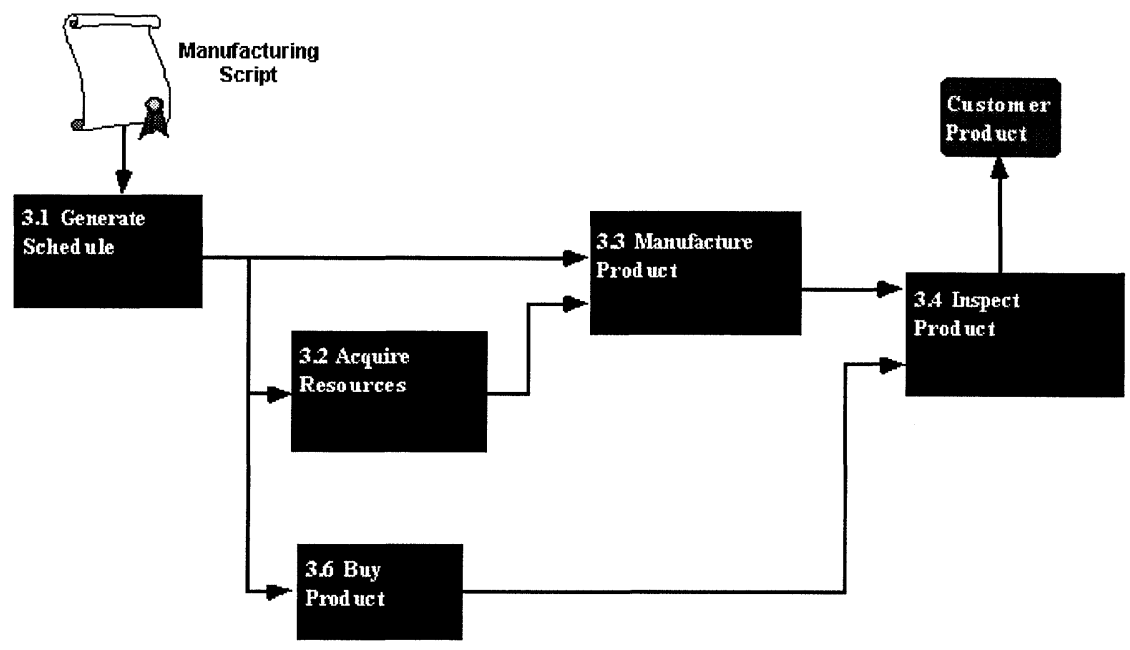

Figure 4 Execution Workflow.

\section{EXECUTION OF THE WORKFLOW}

Integrated product realization demands a control mechanism. With the increasing importance of geographically distributed enterprises, TEAM is developing Webbased tools to manage this extended enterprise. Internet access to tools and information is a must in managing today's distributed, agile, virtual enterprises. Internet access provides multi-platform support that allows diverse users to participate in a distributed enterprise using their existing computer hardware, software, and networks. Therefore, the focus of TEAM integration activities for the September 1997 integrated material removal demonstration was the integration of this environment. The demonstration featured implementation and execution of the integrated TEAM product realization process for a General Motors V8 engine cylinder head.

This implies that an environment must be created that integrates all the tools in a process workflow that understands the concept of "attributes." Features of this integrated environment include:

- the tools required to execute the workflow must all interoperate;

- all data conversions must be done in the background, without user intervention;

- a common user interface (for the workflow but not necessarily for each individual tool);

- the ability to link requirements to product attributes;

- the ability to execute any part of the workflow from any location. 
The user starts by defining the process and its attributes. A process is made up of a series of tasks and transitions between the tasks and must be presented graphically to users. For each task, the following information must be entered:

- who can perform the task (based upon qualifications and access to resources);

- inputs (may be files and/or attributes);

- outputs (may be files and/or attributes);

- resources to transform inputs into outputs;

- transitions to other tasks.

Based on the identified customer needs, requirements are captured. Requirements may be updated during execution of the product realization process as necessary. Based on experience and engineering judgment, the user selects product attributes that are needed to evaluate the product design. Some product attributes are relatively simple, such as dimensions, tolerances, and material types. Other product attributes are summary values from complex analyses, such as maximum stress in the part. Once selected, the user enters the product attributes into the enterprise knowledge system. It is expected that product attribute values will be updated throughout the product realization process as an optimal design is determined by iterations through the process. Updated values may also be imported from resources invoked to perform design tasks. In addition to the product attributes, each task in the TEAM workflow model has process attributes that must be defined and entered. Input process attributes represent the information needed by a task to perform the required task functions. Output process attributes can feed downstream tasks in the workflow model or provide process characterization data used for future jobs. After requirement, product, process, and user information is populated during execution of the product realization process, the baseline script and manufacturing script information are available for review, revision, and subsequent execution.

\section{THE FRAMEWORK}

Integrated product realization means that all of the tools in the total manufacturing effort work together in a "seamless" fashion where all information is available in the right form to all applications that need it. In the perfect solution, all systems and tools would comply with universally accepted standards. This is not the case today, nor is it soon likely. Therefore, we must adopt an integration framework as a liaison between all of the different systems and multiple protocols which allows our tools to communicate. An integration framework is a software infrastructure (common objects, common services, and interfaces) that creates a common environment for integrating applications in "plug and play" fashion and sharing information across different computing platforms and operating systems. "Wrappers" and translators associated enable diverse tools to interoperate within 
the enterprise. TEAM has provided such an integration framework for its product realization process with the Web Integration Manager and the Conceptual Design cockpit as discussed below.

\subsection{Web Integration Manager}

Integrated product realization requires a control mechanism to manage the product realization process over the Internet based on enterprise product, process, and resource information. For TEAM, the mechanism must manage the completion of tasks comprising the Concept Optimization, Design Optimization, and Execution phases of the TEAM product realization process to ensure access to consistent and up-to-date design and manufacturing information. As tasks are performed, the script is developed and information is fed back into the enterprise. All information necessary for every task is managed in a secure and controlled environment.

The Web Integration Manager (WIM) was the focus of TEAM integration activities for the September 1997 integrated material removal demonstration. The demonstration featured implementation and execution of the TEAM product realization process for a GM V8 cylinder head under control of the WIM. The WIM provides comprehensive Web-based management of requirements, product and process attributes, resources, and users. It coordinates execution of the defined process by providing elements of workflow and by invoking tools to perform each step in the process. Any process, using any tools, can be modeled and managed by the WIM. From this point forward, however, for example purposes it is assumed that the defined process is the TEAM product realization process as defined by the workflow models presented in Section 1.

For the September 1997 integrated material removal demonstration, TEAM moved from file-based integration to attribute-based integration. Attribute-based integration allows requirements, product models, and process models managed by the WIM to be linked to information managed by resources external to the WIM. For example, WIM product model information is currently linked to selected Pro/E dimensions. This enables tighter integration than is possible with file-based integration. Linkage of requirements to product and process attributes is achieved since all of the information is managed in one application. Since it is not realistic to eliminate files as a mechanism to share information between diverse applications invoked by the WIM, the WIM does provide file storage and retrieval capabilities needed to execute process tasks.

\section{Requirements}

The user can enter requirements directly into the WIM, which provides a logical hierarchy of requirements. It is also possible to import requirements from a requirements management tool, although this requires the integration of the WIM and the specific tool. Similarly, requirements created in the WIM can also be exported to a requirements management tool. Requirements entered directly into the WIM may be updated during the product realization process. 


\section{Product attributes}

Product attributes are used to define the subject part. Typical attributes include dimensions, tolerances, material types, and performance parameters such as cost and miles per gallon. The WIM provides a hierarchical organization of product attributes. Like requirements, product attribute values may be entered directly into the WIM by the user. These values may also be imported from and exported to external resources such as CAD systems. Again, this requires the integration of the WIM and the particular external resource.

Many product attribute values will be updated throughout the product realization process as the design is optimized through multiple iterations. These updates may be performed directly by the user via WIM input screens. Updated values may also be imported from resources invoked to perform design tasks.

\section{Process attributes}

A process consists of a series of tasks and the transitions between the tasks. The WIM provides a hierarchical organization of process steps to support navigation through the process. Each WIM task has associated with it who can perform the task, inputs needed to perform the required task functions, outputs fed to downstream tasks in the workflow model, and resources used to perform the task functions.

\section{User information}

Users must request access to the WIM using the Create_User WIM service. User input includes a login username and password and administrative information such as an e-mail address for notifications. After a user's access request is authorized, he or she may then $\log$ in to the WIM using their specified username and password.

\section{Execution of the Process}

After requirement, product, process, and user information is captured in the WIM, the WIM can be used to coordinate and manage execution of the product realization process. Users $\log$ in to the WIM and select the specific project to be reviewed or updated. Requirement, product, and process information can each be reviewed in detail. A list of pending tasks that the user is expected to complete is maintained based upon the completion of previous process tasks and task transitions. To participate in the process, the user selects a task from his or her list.

For each task performed in the process, the following steps occur:

- When a task is selected, all defined task inputs (files and attributes) are delivered to the user.

- The user invokes resources from the list of defined task resources and uses the resources to complete activities for the task.

- When the user indicates completion of the task, 1) all defined task outputs (files and attributes) are delivered to the WIM for use in downstream tasks, 2) pending task lists are updated based upon tasks that can now be initiated, 
and 3) users whose pending task lists are updated are notified that they can now initiate a task.

When the entire process is completed, the baseline script and manufacturing script information reside in the WIM and are available to the enterprise for execution.

\subsection{Web Aided Engineering}

A reality in moving from the way we do business now to the way we will do business in the future is the challenge of integrating existing "legacy" systems. In the TEAM process, tools are provided which interface with systems not readily compatible with current-generation "open" hardware and software.

Web Aided Engineering (WAE) integrates services with the WIM by linking WIM information to information managed by legacy systems. WAE translators, or "gateways," provide access to the legacy systems without requiring users to have those systems loaded on their machines. WAE gateways take advantage of the robustness of those systems, turning them into WAE services. For the September 1997 integrated Material Removal demonstration, WAE gateways were created for Pro/E, the FElt stress analysis tool, the CostAdvantage cost and manufacturability analysis tool, and the Sandia National Laboratories DAKOTA optimization tool, each of which was featured in the Concept Optimization phase.

When a WAE gateway from the WIM is created in conjunction with a "wrapper" (software that provides a defined interface to access services) around the legacy system, the WIM user has access to needed legacy system information without having to navigate screens associated with that system. This enables automation when coupled with front-end user interfaces to the WIM. A gateway and a wrapper are both required to provide import/export of legacy system information to and from the WIM.

\subsection{Conceptual Design Cockpit}

Integrated product realization, with its systematic approach to manufacturing, opens the door for knowledge-based systems and automated information generation. TEAM has focused its knowledge automation efforts, as a first step, on the Concept Optimization phase with a "cockpit" for conceptual design. The cockpit allows the customer to make choices about what he or she wants and then quickly and "automatically" see the results of those choices.

Where WAE services extend the back-end capabilities of the WIM, cockpits extend the front end. A cockpit is simply a specialized user interface to the WIM that enables users to easily perform some subset of process activities in an automated manner. For the September 1997 integrated Material Removal demonstration, a Conceptual Design cockpit (Figure 5) was provided to allow a single conceptual designer to perform iterative design tradeoff studies in real time using the Conceptual Design tools listed in the previous section once the stress analysis, cost analysis, and optimization problems were defined and set up by domain experts. When used with WAE gateways, cockpits provide a high level of automation. 


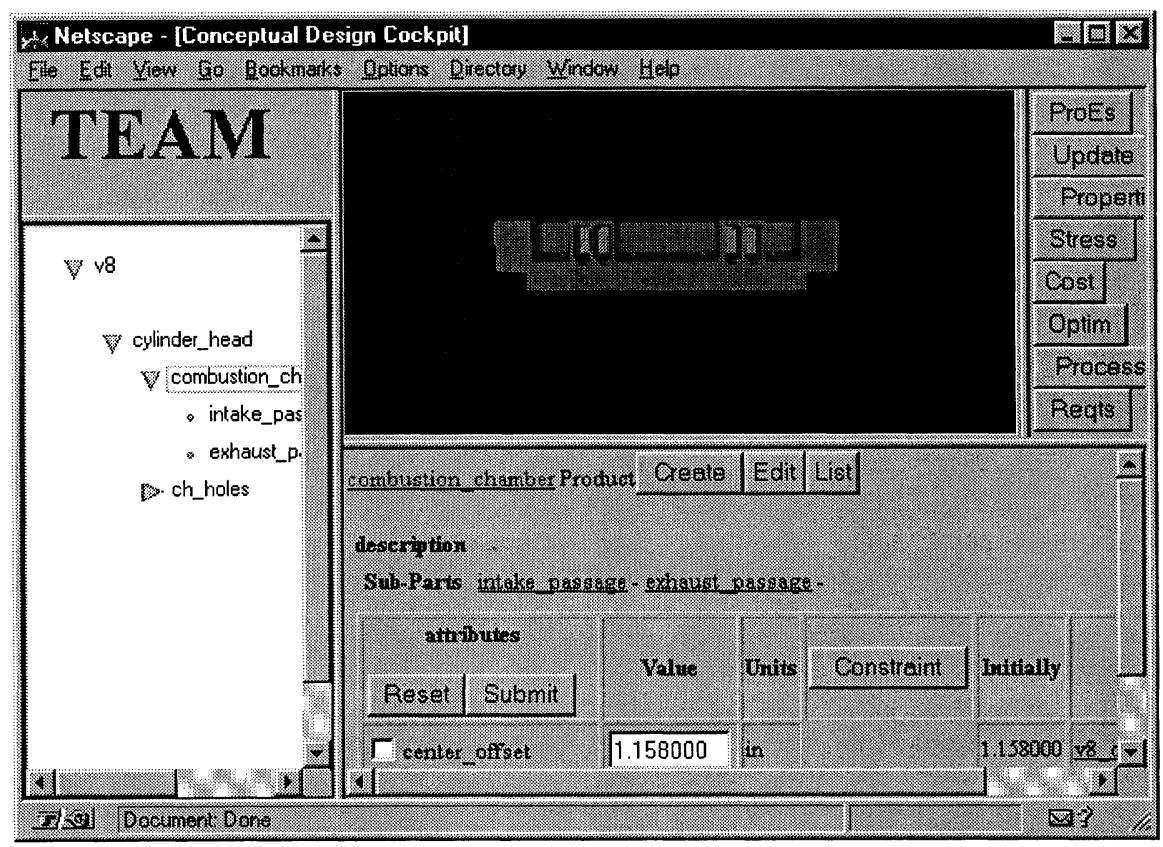

Figure 5 Conceptual Design Cockpit.

\section{NEXT STEPS}

In 1996, TEAM demonstrated the use of product realization and workflow models for manufacturing. In 1997, we added a richer toolset and integration. There is still much to do as we pursue the goal of building a rich manufacturing technology infrastructure that will serve us well into the next century. In 1998, each of the participating Department of Energy TEAM sites will apply TEAM tools and processes to local projects and will present demonstrations highlighting the projects and the impact of TEAM program deliverables on successful completion of the projects.

The Oak Ridge Y-12 Plant will apply TEAM tools to a project featuring the production of a mastering part for dimensional inspection and scheduling of other products manufactured in the same facility. Project tasks comprise a subset of the TEAM product realization process described in Section 1. Processes to be addressed include turning, milling, and dimensional metrology. Production activities including product design, process planning, simulation, numerical control part program creation and verification, detailed work instructions, machining, and inspection will be performed for the mastering part. Shop floor control activities including schedule forecasting and consolidation, tracking of 
individual products along their routing, and documentation management for shop floor activities will also be demonstrated.

The demonstration will be achieved in an integrated environment, supported by the WIM. WIM enhancements to be delivered in support of this project include migration to a Windows 32-bit platform, integration with a commercial objectoriented database, configuration management and access control enhancements, support for tracking and scheduling of multiple products, and an improved WIM modeling user interface. Integration with a project planning tool will facilitate cost, schedule, and resource availability tracking.

\section{REFERENCES}

Neal, R.E., Technologies Enabling Agile Manufacturing Strategic Plan, Oak Ridge Centers for Manufacturing Technology Technical Report, Oak Ridge, Tennessee, 1995.

\section{BIOGRAPHY}

C.K. Cobb (M.S. Computer Science, University of Tennessee) is a Computing Specialist in Lockheed Martin Energy Systems' (LMES) Data Systems Research and Development Division. He is currently the Enterprise Integration Thrust Area Leader for the Technologies Enabling Agile Manufacturing (TEAM) program, a DOE Technology Transfer initiative. Prior to joining the TEAM program, he designed and implemented an electronic document management system that emulates the traditional engineering drawing life cycle and he led a project to electronically capture and index employee medical records. Additionally, he represented LMES during a software development assignment at the International Thermonuclear Experimental Reactor San Diego site. 